\title{
¿CUÁLES SON LAS CONSECUENCIAS DE UNA REFORMA DEL SECTOR ELÉCTRICO DE COSTA RICA? ${ }^{1}$
}

\section{WHAT ARE THE CONSEQUENCES OF A REFORM OF ELECTRIC SECTOR OF COSTA RICA?}

\section{Roberto Jiménez Gómez*}

\begin{abstract}
RESUMEN
De acuerdo con los posibles escenarios de reforma del sector eléctrico las implicaciones serían: a. El Instituto Costarricense de Electricidad (ICE) dejaría de ser el responsable del suministro eléctrico, pasando a las distribuidoras; b. Se perdería la planificación normativa de largo plazo; c. Se deberían eliminar las empresas verticalmente integradas; d. ICE dejaría de ser el comprador único en el mercado nacional y el Mercado Eléctrico Regional (MER); e. El ICE dejaría de tener el Centro de Despacho del Sistema Eléctrico Nacional (CENCE), a su cargo; f. Se daría una reducción de la generación con fuentes renovables.
\end{abstract}

PALABRAS CLAVES: COSTA RICA * ENERGÍA ELÉCTRICA * REFORMA ECONÓMICA $*$ MERCADO * ESTADO * MARCO INSTITUCIONAL * GENERACIÓN DE ENERGÍA * CONSECUENCIAS SOCIALES * RECURSOS RENOVABLES

\section{ABSTRACT}

According with the possible stages of the reform of the electric sector in Costa Rica, the consequences that would be considered are: A. ICE would not be the solely responsible for the electric supply, this responsibility would pass to the distribution companies. B. Normative long-run planning would be lost. C. Vertically integrated firms should be eliminated. D. ICE would not be the only wholesale buyer in the national and MER markets. E. ICE would not has the responsibility for the centralized generation dispatch. F. Renewable sources are reduced.

KEYWORDS: COSTA RICA * ELECTRIC POWER * ECONOMIC REFORM * MARKET * STATE * INSTITUTIONAL FRAMEWORK * POWER GENERATION * SOCIAL IMPLICATIONS * RENEWABLE RESOURCES

Centro Internacional de Política Económica, CINPE, Universidad Nacional e investigador de un proyecto en la Vicerrectoría de Investigación de la Universidad Estatal a Distancia

RJimenezG@ice.go.cr
1

Este artículo forma parte de la investigación de mi tesis doctoral en Gobierno y Políticas Públicas de la Universidad de Costa Rica denominada: "Análisis del proceso de formulación de políticas para la reforma del sector eléctrico y su potencial incidencia en el marco institucional y la producción con fuentes renovables". 


\section{INTRODUCCIÓN}

El presente trabajo presenta la discusión sobre las reformas económicas neoclásicas, planteando la problemática de la función y alcance del Estado y el mercado. A partir de las reformas neoclásicas realizadas en los sectores eléctricos de América Latina, establece las principales lecciones aprendidas, para luego analizar de forma general, el proceso de reforma que ha estado en la agenda pública en Costa Rica.

Con base en un estudio basado en el análisis de redes de política pública y mediante escenarios de prospectiva se determinó el tipo de reforma posible para el sector eléctrico en Costa Rica. Con base en ello se establecen las principales implicaciones sobre el marco institucional y la generación con fuentes renovables.

\section{LAS REFORMAS ECONÓMICAS}

Los procesos de reforma económica han sido un común denominador en cerca de tres décadas en el ámbito internacional. Para los países en vías de desarrollo diversos factores afectaron su situación económica, llevando a la crisis de la deuda externa de inicios de la década de 1980. La crisis llevó a tener que aplicar políticas de reforma bajo las directrices de los Organismos Financieros Internacionales (los OFI), además de pagar las deudas a los diferentes acreedores, para poder acceder a préstamos.

Agudizaron o complementaron los problemas y la ejecución de reformas económicas en las décadas de 1980 y 1990, situaciones como las siguientes: a. Las debilidades y agotamiento del modelo keynesiano aplicado (Ffrench, 1988); b. Los problemas del modelo de industrialización por sustitución de importaciones en los países latinoamericanos; c. Préstamos de corto plazo y bajo condiciones desfavorables brindados a finales de los setenta e inicios de la década de 1980, llevaron a modificar sus condiciones, lo que condujo a desequilibrios financieros de los países en vías de desarrollo; d. El reposicionamiento de sectores políticos conservadores en países desarrollados de gran influencia que criticaban el papel excesivo del Estado y pregonaban la iniciativa privada $y$ el mercado como las soluciones a los problemas de la época (Stiglitz, 2003b; Krugman, 2000).

Siguiendo la argumentación de Stiglitz (2003b), los conservadores encontraban que los costos de la regulación eran elevados. El propósito de las políticas reguladoras era que a través de medidas administrativas como: normas, requisitos y reglamentos que buscan moldear la conducta de los agentes privados, beneficiaran al interés público. Sin embargo, estas medidas recibieron importante crítica por la regulación excesiva e innecesaria, dando la sensación de que algunos costos aumentaron en la década de 1970 por esas razones.

En cuanto a la regulación de la competencia, generalmente los monopolios naturales se dejan en empresas públicas. En Estados Unidos de América (EE.UU) se permitió a las empresas privadas con regulaciones de precios máximos, y niveles mínimos de servicios. En la década de 1980, como lo indica Krugman (2000), los conservadores dieron su acto de fe al poder del libre mercado, pasando de monopolios naturales a empresas privadas no reguladas, lo cual resultó con efectos negativos sobre la sociedad.

Para Krugman (2000), los procesos de regulación de la economía, en países desarrollados como EU, se fue haciendo cada vez más compleja. El proceso de regulación comenzó a mostrar más interés en proteger las industrias, que el de los consumidores. Los defensores del libre comercio ganaron la batalla fácilmente. La tesis básica fue: la regulación creciente a que estaban sometidas las empresas era un lastre para el crecimiento económico. Por ello se culpó a la regulación por la desaceleración de la productividad. Esto tomó en cuenta el contexto de la nueva legislación en la década de 1970 en materias tales como nuevas reglamentaciones para proteger la salud de los trabajadores, la seguridad de los consumidores y la protección del medio ambiente.

Los procesos de desregulación económica llevados a cabo inicialmente en los Estados Unidos, entre las décadas de 1980 y 1990, influyeron en su adopción en el resto del planeta. Para Stiglitz (2003b), esta visión llevó a la burbuja del exceso de inversión en telecomunicaciones en el 2001, a la manipulación del mercado eléctrico 
en California y a los conflictos de intereses en el sector bancario, entre otros ${ }^{2}$. Para este economista lo que se requería era una reforma de la regulación, no una desregulación, lo cual llevó importantes costos para la economía.

Los costos de regulación y dirección por parte del Estado, así como las críticas a cualquier sistema de subsidios sociales, influenciaron en gran medida en los procesos de reforma económica en diferentes sectores del ámbito internacional, pero sobre todo en los países en vías de desarrollo, por al menos dos razones. Por una parte, la relación de pesos y contrapesos en los países desarrollados llevó, como en el caso británico, a que pese a la visión de las reformas, gran parte de los programas sociales se mantuvieran y los cambios no fueran tan ortodoxos como convencionalmente se ha querido mostrar (Alcantara, 1995).

Otro elemento coyuntural fue que los programas de ajuste estructural que fueron originalmente usados para los procesos de reforma, se efectuaron en condiciones de fuerte condicionalidad cruzada de los organismos financieros internacionales, en donde los desequilibrios macroeconómicos de estos países en vías de desarrollo les daba pocos grados de libertad para poder negociar y plantear políticas de ajuste de acuerdo con las características de sus países y objetivos nacionales.

Los planteamientos que sustentan la reforma han estado apoyados en el plano internacional por un conjunto de intereses económicos que han accedido al poder político en los principales países del mundo, así como a organismos internacionales, haciendo que sus postulados lleguen a dominar de forma abrumadora en todas las esferas de la vida social en el planeta ${ }^{3}$.

Bajo ese contexto, se han estado dando los procesos de reforma económica en el ámbito internacional, con una orientación del pensamiento conservador en lo político y el enfoque

Estos aspectos adquieren más importancia en la segunda mitad del 2008, con las graves consecuencias de la crisis inmobiliaria en los Estados Unidos y la quiebra de los principales bancos de inversión de ese país, llevando a la mayor crisis económica en los últimos 50 años. monetarista en lo económico. La discusión está abierta, la crisis del sistema financiero internacional, los problemas del crecimiento y desempleo que se han planteado a partir de ello y las propuestas de políticas keynesianas de Obama y la necesidad de llevar a cabo una revisión de los paradigmas dominantes planteado por la CEPAL ${ }^{4}$, establecen un contexto de discusión y redefinición de la relación que debe existir entre el Estado y el mercado.

La justificación de un Estado que no sea el mínimo se ha planteado con diferentes argumentaciones. Por una parte, existe una dimensión ética en el sentido de que debe mantenerse un Estado social de derecho que permita la inclusión de un conjunto de derechos fundamentales en el plano económico, social y cultural, la satisfacción de ciertas necesidades básicas y el acceso a ciertos bienes fundamentales para todos los miembros de la sociedad. Otra justificación es la necesidad del Estado en el sistema capitalista de ser un garante de la distribución del ingreso para efectos de poder tener demandantes de bienes y servicios, eso incluye el ser demandante al mismo Estado en el sistema (Cortina, 1998).

Los procesos de reforma económica y del Estado no son neutrales, beneficia a unos y perjudica a otros. Una serie de políticas públicas en los últimos 25 años han estado orientadas a fomentar ciertas actividades económicas, estableciendo de partida ganadores de las reformas y redistribuyendo el excedente económico de la sociedad.

"Los informes y estudios del FMI y del Banco Mundial o de la OCDE se imponen sin competencia, ofreciendo datos económicos y estadísticos, indicadores de crecimiento y modelos de desarrollo. Estas instituciones tienen equipos de investigadores $y$ redes de expertos cuya visión del mundo es análoga, siguen los mismos procesos de formación, están condicionados por el mismo molde ideológico y disponen de instrumentos técnicos de prestigio considerable”. (De Senarclens, 2001: 12).

4 Ver: <http://media.eclac.cl/presentaciones/de/ balance2008/balance2008.html>

Bárcena, A. Balance preliminar de las economías latinoamericanas. CEPAL, 18 de diciembre 2008. 


\section{EL ESTADO Y EL MERCADO}

Los críticos de las reformas económicas neoclásicas consideraban que para que se puedan desarrollar los mercados es necesario que existan un conjunto de condiciones, en donde es precisamente el Estado, como representante del interés general, el que debe paulatinamente ir definiendo $y$ desarrollando las condiciones para que el sistema económico y las formas de convivencia social cuenten con un marco institucional.

Un problema fundamental en los procesos de reforma llevados a cabo por los OFI, ha sido que estas han creído que automáticamente se tendrán las condiciones para que surjan mercados que funcionarán bien. Lamentablemente el mercado como institución histórica y social ha tenido fuertes deficiencias estructurales en países como los latinoamericanos, lo cual ha llevado a que las reformas no hayan tenido los resultados buscados. Unido a ello se debe tener presente que la orientación básica ha sido ver al Estado como un problema, lo cual ha conducido a su desmantelamiento y pérdida de capacidades para regular, fiscalizar y orientar las reformas, lo cual ha tenido resultados negativos en muchos países.

En ese sentido es importante retomar el aporte de North (2005). Para él es claro que las instituciones surgen para reducir la incertidumbre presente en los procesos económicos, una vez que empiezan a adquirir algún grado de complejidad. Estas existen por la limitación de los procesos humanos de computación frente al desarrollo de la información y por la existencia de costos de transacción en todos los ámbitos de la interacción humana (Valdivieso, 2001).

Justificante y complementario a lo anterior, está el hecho de que las instituciones en los países en vías de desarrollo no han progresado adecuadamente, su capacidad para cumplir sus funciones es limitada por razones históricas, sociopolíticas, recursos o bien por la misma cultura existente.

Se reconocen aspectos históricos, culturales, sociopolíticos y económicos que influyen en la forma de organización social, en la manera como los agentes individuales y colectivos toman sus decisiones de consumo, inversión y de convivencia social. El pensar que con más intervención del Estado o más mercado, sin comprender la realidad de cada país, en sus diversas e interrelacionadas dimensiones, ha llevado a tomar decisiones de política pública e incluso modelos económicos y sociales que no son viables en el contexto latinoamericano o bien sus resultados no son los deseados.

Los procesos de privatización en términos generales han dejado de lado el contexto, las características estructurales de los países en el ámbito social, económico y político. Por otra parte se han tenido tesis que señalan casi como imposible que puedan existir empresas públicas eficientes, que tengan funciones estratégicas para propiciar condiciones para el desarrollo, en donde la empresa privada no necesariamente puede hacerlo o bien los costos de transacción por la regulación son elevados debido a su complejidad.

En general, las décadas de 1980 y 1990 han estado dominadas por una orientación hacia el libre mercado, la empresa privada y la menor participación del Estado. Esto ha ido creando una dicotomía que para muchos es falsa con la regulación inteligente y racional de los mercados, la participación pública en áreas estratégicas y la orientación de políticas públicas discrecionales que promuevan el desarrollo $y$ disminuyan las desigualdades sociales.

El mercado y el Estado son dos componentes claves del marco institucional y no necesariamente están en conflicto. La descontextualización histórica, social, política e institucional ha llevado a la recomendación de reformas económicas muy similares en países con sociedades distintas, con instituciones de diferentes características y capacidades, por lo cual los resultados y los efectos de estos procesos no han sido los esperados.

Se debe tener presente que tanto el Estado como el mercado tienen limitaciones, debilidades y fallas. La búsqueda de políticas públicas basadas en análisis rigurosos de la realidad, que permitan adoptar inteligentes combinaciones de instrumentos en donde el mercado $y$ el Estado se complementan $y$ desarrollan capacidades institucionales. Lo anterior con el fin de que cada uno de ellos pueda cumplir 
una función que permita el logro de los objetivos de política nacional que lleven al mayor desarrollo, son elementos que deben prevalecer (Germa, 2004).

El mercado y el Estado no tienen por qué ser contradictorios, en el contexto de América Latina se deben generar complementariedades entre uno y otro, ya que la realidad social, política, económica e institucional es compleja, requiriendo crear las condiciones a mediano y largo plazo para mejorar el bienestar de la mayoría de la población (North, 1993).

Dentro del desarrollo del sistema capitalista, el mercado ha tenido un papel básico, requiriendo un proceso de depuración y de desarrollo histórico. El mercado es una institución histórica que se ha ido construyendo y a la cual los mismos Estados han tenido que ir estableciendo ajustes normativos con el fin de que puedan contribuir a ciertos fines de una colectividad, mejorar la asignación de los recursos y no generar poder excesivo de determinados agentes. Los errores, ajustes e intervenciones por parte de los Estados desarrollados sobre el mercado a través de muchos años son elevados, mostrando que pese a que podrían ser un buen asignador de recursos en ciertos sectores, ellos no se crean de la noche a la mañana (Stiglitz, 2002).

El dilema es cómo compatibilizar la mayor eficiencia del mercado que indican sus defensores con la equidad, así como la acción del individuo racional y egoísta, con ciertas orientaciones básicas y necesarias que aseguren la reproducción social que brinde equidad. En qué medida por tanto, el conflicto entre el Estado y el mercado existe, o bien hay espacios en donde se puedan definir funciones $y$ potestades a ambos que permita un marco de convivencia social que beneficie a la colectividad y promueva el desarrollo. Estos aspectos son centrales para el diseño de una posible reforma del sector eléctrico en Costa Rica.

En la relación mercado y Estado las diferencias mayores se encuentran en muchos casos en elementos relacionados con la gradualidad, el diagnóstico adecuado y los riesgos que ocasiona la incertidumbre cuando los mercados están liberados (sector financiero), por la movilización especulativa de los recursos.
La discusión entre privatización, desregulación, mercado y Estado ha dejado de lado que se requiere de un marco institucional básico y que la capacidad de gestión del Estado y las organizaciones, es decir en un sentido amplio la institucionalidad, constituyen elementos centrales, para que una economía funcione bien.

Posterior al desmantelamiento de las organizaciones del Estado en muchos países, ha surgido por lo general, la necesidad de contar con leyes, normas, organizaciones que funcionen bien y orienten las relaciones de convivencia social. Ello ha llevado a la necesidad de fortalecer las organizaciones para que el Estado, el mercado y la democracia política, sean efectivos medios para promover el desarrollo y la equidad social.

Los resultados obtenidos, las desigualdades económicas y sociales, así como complejos procesos sociopolíticos, llevan a cuestionar las reformas económicas, dentro de este contexto se tienen al menos dos opciones: a. Retroceder en los procesos de privatización y liberalización $y$ volver a los modelos estatistas; b. Efectuar los ajustes a los sistemas reformados con una mayor rectoría, regulación, participación cuando sea conveniente y además de la supervisión del Estado, como promotor de un desarrollo de las instituciones con reglas del juego que permitan el desarrollo del mercado, con un papel más activo del Estado. Este es un reto de la sociedad contemporánea.

El Estado debe tener un papel importante en la redistribución del ingreso, porque el enfoque del goteo ha mostrado sus limitaciones y ha llevado en la mayoría de los casos al empeoramiento de las condiciones sociales, de la distribución del ingreso, de la calidad de vida $y$ del bienestar de importantes sectores de la población de los países en desarrollo.

La búsqueda de un equilibrio entre el Estado y el mercado, para que el primero adquiera nuevas competencias en ciertos campos, según señala esta perspectiva, supone abrirse al juego del mercado de forma acotada, de manera que este pueda contar con las condiciones para poder funcionar mejor, creando las bases que permitan un desarrollo y perfeccionamiento de su accionar. 
El sector eléctrico tiene una serie de particularidades que restringen y limitan la creación de mercados competitivos que funcionen bien. Por otra parte, junto con la creación de los mercados, el Estado a través de leyes, normas, directrices, organizaciones con buena capacidad de gestión e independencia, son básicos.

Es por ello que antes de pensar en procesos de reforma económica en América Latina, tal como la privatización o desregulación de sectores estratégicos como el eléctrico, al margen de otras consideraciones, se debió analizar las instituciones que se tenían y fortalecerlas con el fin de que un sistema económico basado en el mercado $y$ empresas privadas tuviera una base institucional más sólida.

\section{CONTEXTO DE LAS REFORMAS DEL SECTOR ELÉCTRICO}

El desempeño que habían tenido los sectores eléctricos de propiedad estatal no habían sido los deseados en algunos países, por ineficiencias internas o por acciones deliberadas, con el fin de disminuir la calidad de los servicios eléctricos. Dentro de las fallas de los sistemas eléctricos preponderantemente de propiedad pública se indican los siguientes: (Millán, 2006) a. La expansión de la generación basada en el costo mínimo usando el potencial hidroeléctrico de Latinoamérica en la mayoría de casos no fue posible, debido a razones como: aspectos técnicos, escasa capacidad de gestión, intereses de regiones $y$ sectores que querían ver su proyecto; b. Las limitaciones de soluciones de mínimo costo debidas a la poca cantidad de proyectos en cartera con suficientes estudios para poder ser considerados; c. Intereses de las empresas proveedoras, junto con corrupción política hacían que los contratos de construcción se encarecieran y por ende el costo de las plantas finalizadas; d. La crisis de inicios de los ochenta llevó a un fuerte debilitamiento de las financieras de las empresas públicas por una parte, las tarifas no crecían según el aumento de los costos, en especial los servicios de las deudas externas y por otra parte, la crisis llevó a disminuciones de la demanda, afectando (capacidad instalada excedente con préstamos por pagar) los ingresos de las empresas en su liquidez y Estados de Resultado de forma significativa; e. El manejo político de las tarifas, junto con las pérdidas técnicas y por robos en las líneas de distribución de parte de la población, fueron aspectos que afectaron seriamente las empresas públicas; f. La falta de incentivos correctos para el estímulo de la eficiencia y mejora de la gestión.

Por ello se puso la esperanza en que los sistemas de propiedad privada, bajo competencia pudieran por fin coadyuvar al anhelado desarrollo de los países de la región. En general se tuvo una gran confianza en que la creación de mercados en el sector eléctrico permitiría importantes beneficios, a través de la competencia que llevaría a mayor eficiencia. Para ello se desarrollan diferentes variantes de modelos para lo que llaman industria eléctrica con el fin de aprovechar los potenciales beneficios de la competencia.

Besant (2006) indica que las razones que justificaron las reformas en el sector eléctrico son: la búsqueda de una mejora en el servicio, el mayor acceso a la electricidad a los pobres y la mejora de la posición fiscal de los gobiernos (generalmente por medio de la venta de activos). Las reformas se basan en la reestructuración de las empresas, la regulación, la competencia y la definición de los roles de los participantes públicos y privados.

Estos procesos en el contexto de los programas de ajuste estructural (PAE) en América Latina son retomados, $y$ llevan a los procesos de privatización, apertura y desregulación en diversos sectores de las economías, uno de ellos fue el sector eléctrico. Dentro del sector energía los sectores eléctricos habían sido operados por empresas eléctricas, generalmente de propiedad estatal, debido al interés estratégico dado a este sector para el desarrollo económico, el carácter monopólico natural que se le asignaba $y$ la influencia del modelo Keynesiano y de la ISI, en caso de América Latina.

Es precisamente en ese sector que se inician las reformas más significativas a finales de 1970 como son los casos de Chile que se usa como experimento y de Gran Bretaña posteriormente. Con los PAE, la inestabilidad macroeconómica en América Latina, los intereses político 
empresariales, entre otras causas, llevan en la década de 1990 a importantes reformas en el sector eléctrico de la región, dirigidas hacia la privatización, creación de mercados y promoción de la inversión extranjera (Pollitt, 2005).

\section{RASGOS FUNDAMENTALES DE LAS REFORMAS}

En el ámbito internacional en la década de 1980 y 1990, se dan los cambios más importantes tendientes a las transformaciones de los sistemas eléctricos. Estas reformas se han justificado por: a. Los cambios tecnológicos, que han permitido reducir la escala de las centrales de generación y una mayor competencia; b. Una revisión de la función reguladora que desempeñan los gobiernos.

Estas reformas han consistido de forma general en lo siguiente (Bazán, 2003; Besant, 2006): a. Se da una desintegración vertical entre las actividades potencialmente competitivas (generación y comercialización) y las actividades reguladas (transmisión y distribución) del sector; b. La introducción de la competencia en la generación y en la comercialización; c. La creación de mercados eléctricos descentralizados cuya característica fundamental son los mercados eléctricos de contado al por mayor (wholesale electricity spot markets) o pools competitivo; d. La descentralización de las decisiones de inversión en expansión de la red de transmisión y en capacidad de generación; e. La regulación de las tarifas y de las condiciones de acceso de terceros a las redes eléctricas (que aún se consideran monopolios naturales); f. Una redefinición de la regulación (desarrollo de un nuevo marco regulador); g. Se han realizado privatizaciones en la generación y en la distribución con la esperanza de que los inversionistas privados introduzcan recursos financieros en estas fases $y$ con la expectativa de que las empresas busquen reducir sus costos de manera que se reflejen en beneficios para el cliente final.

Jamsb (2006) presenta una síntesis de los procesos de reforma realizados en el ámbito internacional. Muestra el camino común que han seguido los procesos de reforma, pasando de la regulación, a leyes de la industria eléctrica, eliminación de las empresas verticalmente integradas, creación de competencia y privatización. La complejidad de los procesos, la dificultad de creación de mercados que funcionen bien, son elementos que hacen difícil el logro de mayor eficiencia y sobre todo que los usuarios finales vean disminuidas las tarifas.

Los procesos de reforma del sector eléctrico en América Latina han tenido por lo general como primer paso la aprobación de una ley de electricidad de carácter general que regula $y$ define las reglas del juego a lo que llaman industria eléctrica. Con estas leyes se busca establecer las normas generales para que los servicios eléctricos pasen a ser bienes o mercancías transables en mercados con segmentos específicos como los grandes consumidores. Una segunda fase generalmente tomado en los procesos de reforma fue el definir un marco regulatorio y si no existía crear o fortalecer la instancia reguladora del país. Destaca de varios países de la región los tiempos tan cortos entre la adopción de las leyes de electricidad, las del ente regulador y los procesos de creación de mercados. En los países en donde la capacidad de gestión, la autonomía política, la cultura institucional e histórica y la dotación de recursos humanos en el ámbito de la regulación eran débiles, resulta difícil pensar que dichos entes pudieran asumir con propiedad los retos de la apertura y la complejidad que significan los sistemas eléctricos que son reformados. Una tercera fase de los procesos de reforma del sector eléctrico se puede llamar la corporalización de las empresas generalmente públicas existentes. Unido a ello la cuarta fase fue la reestructuración de las empresas, que consistió en la división según la cadena de valor, separando producción, distribución y transmisión, además de dividir estas en empresas de generación y distribución bajo ciertos criterios. La fase quinta se puede ubicar en algunos países con la creación de competencia en un mercado mayorista $y$ de contratos, para luego en la fase sexta llevar a cabo privatizaciones, acción que en algunos casos se realiza antes de empezar a crear competencia en los mercados mayoristas 
$y$ de contratos. Se podría ubicar una sétima fase que en muchos casos consistió en la depuración de los mecanismos de creación de mercados; incentivos, regulaciones específicas, ajustes a la regulación en algún ámbito.

La secuencia de reformas predicada por la documentación sobre el tema comienza con el establecimiento de un marco regulador sólido, continúa con la reestructuración de los activos públicos y la organización de los mercados y finaliza con la privatización, primeramente por el segmento de distribución (Millán, 2006: 59).

En el caso de América Latina, los procesos de reforma del sector eléctrico corresponden al auge del Consenso de Washington, en donde la aplicación total de dichas directrices fue ejecutada en este sector. Resalta la rapidez con que la mayoría de países realizaron las reformas más importantes, sin considerar las especificidades, carencias y dificultades de ajuste rápidos. Asimismo, mostraron las debilidades que una reforma estructural, como la realizada, podría tener sobre las señales de inversión de los agentes económicos, las fuentes energéticas, el ambiente y los sectores sociales más vulnerables.

\section{LA DISCUSIÓN ACTUAL SOBRE LAS REFORMAS DEL SECTOR ELÉCTRICO}

En el ámbito internacional la mayoría de las reformas del sector eléctrico se realizaron en la década de 1990. Los resultados obtenidos de estos procesos varían de acuerdo con el país o la región que fue sujeta de esos cambios. Se aprecia que en términos generales los países europeos han ido implementando reformas de corte neoclásico de forma paulatina, sostenida, en tiempos más amplios, en donde los resultados en términos generales no parecen llevar a retroceder en los procesos hacia un enfoque de mayor participación del Estado, pero sí de análisis y ajustes. La discusión en Europa pasa por mejorar los diseños institucionales y las reglas del juego, con el fin de depurar los sistemas eléctricos, disminuyendo o eliminando las deficiencias y problemas que han mostrado los sistemas reformados. Hay aspectos sustantivos que todavía no han sido resueltos como la compatibilización de una lógica de la competencia en los mercados; con una efectiva $y$ no discriminatoria (sobre todo en la red eléctrica) de las fuentes renovables de energía eléctrica, así como el poder que algunos agentes tienden a tener en los mercados.

En el caso de los Estados Unidos, los problemas presentados en California llevaron a una revisión de ciertos aspectos específicos de los diseños de los mercados, con el fin de mejorar $y$ con ello prevenir nuevos problemas. En ese sentido la depuración y mejoramiento de los mercados es el camino que de forma general ha seguido este país.

"En los países latinoamericanos no ha sido fácil establecer mercados eléctricos competitivos $y$ atraer al mismo tiempo las inversiones necesarias para asegurar la suficiencia de la oferta eléctrica para atender la demanda" (Millán, 2006: 102).

América Latina se encuentra ante el reto de estructurar mercados creíbles y adecuados, en donde los precios jueguen su papel, que existan incentivos para las inversiones en nuevas plantas de generación eléctrica, en especial renovables, junto con la mejora en las instituciones y reglas del juego, son temas centrales, pero en muchos casos, aún pendientes en el sector eléctrico; incluso en los países que han tenido un mejor desempeño y experiencias con las reformas del sector eléctrico.

En el caso del sector eléctrico chileno, el primero en reformarse, ha sido constante en los procesos de reforma, no sin antes tener problemas de abastecimiento y cuyo reto es cómo incorporar fuentes renovables en la generación eléctrica. Recientemente se ha tratado de modificar el enfoque de corto plazo, además de tratar de promover las fuentes renovables, mediante instrumentos diferentes a la lógica misma del mercado, a partir de orientaciones de política pública. En el caso de El Salvador en donde se aplicó uno de los modelos más puros de mercado, se han tenido que realizar modificaciones legales, tratando de solucionar una serie de problemas importantes que se han dado. 
La preocupación por el doble papel del Estado como regulador y definidor de políticas resulta válida, sin embargo, en el contexto de la región, ha sido más corriente el uso de la regulación y la definición de políticas para extraer rentas para determinados actores sociales, generalmente empresarios, en los sectores como el eléctrico. En ese sentido contrario a lo indicado por Millán (2006), los cazadores de renta se ubican en diferentes ámbitos y solamente un marco institucional fuerte puede minimizar este aspecto, no teniendo que ver con la propiedad de los activos.

Por otra parte, ante la falsedad de la dicotomía entre Estado y mercado y la problemática energética internacional, ha vuelto a surgir el criterio de que es posible que el Estado pueda continuar realizando actividades de forma directa, en ámbitos y proyectos en donde el sector privado no puede o no desea hacerlo, o bien el sector público ha mostrado la capacidad para hacerlo bien, dándole al sector privado el espacio para participar en igualdad de condiciones con el sector público. Los diseños de las reformas que consideren estos espectros más amplios son parte de las discusiones actuales.

\section{LAS PRINCIPALES LECCIONES DE LOS PROCESOS DE REFORMA}

Dentro de los problemas encontrados según el tipo de diseño de las reformas y la realidad de cada país están los siguientes:

a) Se ha indicado que el mercado eléctrico por sí solo es insuficiente para propiciar las inversiones en proyectos de generación eléctrica con fuentes renovables, situación que enfrentan la mayoría de países que han llevado a cabo procesos de reforma (CEPAL, 2004).

b) La solidaridad social hacia los sectores de menos ingresos no se ha dado adecuadamente en los países en donde se han llevado a cabo reformas, lo cual se evidencia por lo general en tarifas elevadas, dificultades de acceso y las zonas no rentables no han sido cubiertas.

c) Estos aspectos plantean un problema serio al proceso de las reformas en el sector eléctrico, lo cual ha sido aún más grave en la mayoría de los países en vías de desarrollo debido a la falta de capacidad de las organizaciones, el incumplimiento de la normativa, la participación de la influencia política y de intereses económicos en la toma de decisiones.

Diversos estudios establecen limitaciones a los sistemas eléctricos reformados, dentro de los aspectos más relevantes están los siguientes (Bazán, 2003; Millán, 2006; Besant, 2007):

$\diamond$ Se puede observar que la competencia (imperfecta) entre las empresas eléctricas depende en gran medida de las reglas de acceso a las redes de transmisión y distribución que se adopten tras la reforma, $y$ de que en el sistema no se presenten problemas de congestión, es decir, que el sector cuente con suficiente capacidad para realizar los intercambios de electricidad entre regiones distantes.

$\diamond$ Si se realiza la reforma del sector eléctrico sin cuidar el correcto diseño de los nuevos mecanismos institucionales que gobiernen el acceso a las redes eléctricas $y$ sin adoptar medidas para reducir la elevada concentración heredada del modelo tradicional, no se conseguirá mejorar los resultados del mercado eléctrico respecto del modelo tradicional.

$\diamond \mathrm{Si}$ tras la reforma del sector eléctrico no existen ganancias de eficiencia productiva (reducción de costos de producción), los consumidores tendrán que pagar precios más altos por la electricidad consumida respecto a los precios del modelo centralizado. No obstante, si la ganancia de eficiencia productiva es lo suficientemente grande, es posible que se produzcan situaciones donde los precios disminuyan tras la reforma del sector.

Dentro de los elementos que han limitado la competencia para el funcionamiento de los mercados en los países en desarrollo se señalan: a. Se ha dado una tendencia a tener una insuficiente capacidad de generación que cubra los crecimientos de la demanda que permita la seguridad de suministro y aumento del acceso de electricidad; b. El poder regular 
mercados con una combinación de fuentes térmicas e hidroeléctricas ha sido especialmente más difícil, que otros sistemas; c. El control del abuso de empresas que tienen un poder de mercado importante ha sido difícil, incidiendo en los precios; d. Las reformas se han llevado a cabo en países en donde el tamaño del mercado es pequeño para soportar un número viable de vendedores y para cumplir con los requerimientos básicos para el desarrollo de un mercado eléctrico competitivo; e. El inadecuado desarrollo de la capacidad del sistema de transmisión de electricidad y del sistema de control de electricidad para administrar sistemas eléctricos más complejos dados los flujos de electricidad que se dan en un mercado competitivo; f. En términos generales en muchos países que se han llevado a cabo reformas ha faltado diversificación de fuentes energéticas para generar electricidad y ofrecerlas en los mercados para que se dé una competencia entre cada una de estas fuentes (Besant, 2006).

Para contar con inversión en el sector eléctrico las privatizaciones de los activos existentes no llevaron a las inversiones adicionales requeridas en fuentes renovables. Para ello era necesario contar con un diseño del sistema eléctrico con reglas claras y orientadas al fomento de las fuentes renovables, además de una buena regulación del Estado y capacidad de gestión de las organizaciones relacionadas con el sector. Lo anterior era relevante pues, en los países en vías de desarrollo por lo general se tienen instituciones débiles, un desarrollo empresarial y económico insuficiente, sectores estratégicos de infraestructura y servicios con importantes limitaciones, además de altas desigualdades distributivas.

Quizás el aspecto central de las reformas de los sectores eléctricos son los mercados mayoristas. Se ha tenido mucho optimismo en su aplicación, pero los resultados han brindado una serie de enseñanzas y problemas que han llevado a configurar diferentes soluciones en los países. Dentro de los principales problemas están: a. La dificultad de contar con diseños del mercado que sean adecuados al contexto del país y de sus objetivos para con el sector eléctrico; $b$. Se ha tendido a que uno o varios agentes económicos logren un ejercicio de poder de mercado para su beneficio; c. Las señales económicas para mantener las inversiones que aseguren el suministro no han sido las mejores; d. Ha resultado difícil el valorar $y$ pagar servicios auxiliares de gran importancia para los sistemas eléctricos; e. El manejo de la congestión de la red de transmisión eléctrica ha sido uno de los problemas difíciles de solucionar (Millán, 2006; Besant, 2007).

En general para que las reformas de mercado en los sistemas eléctricos puedan brindar más beneficios que costos, deben permitir crear mercados competitivos, lo cual está determinado por un conjunto importante de condiciones y características, no fáciles de lograr. Si un sistema eléctrico reformado no puede lograr sistemas competitivos que estimulen fuertemente la eficiencia, es posible que no compensen los beneficios de los sistemas verticalmente integrados y planificados normativamente, como el sistema eléctrico de Costa Rica. 
TABLA 1

ORGANIZACIONES DEL SUBSECTOR ELÉCTRICO SEGÚN ACTIVIDAD O COMPETENCIA QUE EJERCEN

\begin{tabular}{|c|c|c|c|c|}
\hline ACTIVIDAD O COMPETENCIA & \multicolumn{4}{|c|}{ ORGANIZACIONES PARTICIPANTES } \\
\hline $\begin{array}{l}\text { Rectoría y definición de políticas } \\
\text { nacionales }\end{array}$ & \multicolumn{4}{|c|}{$\begin{array}{l}\text { El Poder Ejecutivo por medio del Ministerio de Ambiente y Energía (MINAE) } \\
\text { sustentado en la Dirección Sectorial de Energía. }\end{array}$} \\
\hline $\begin{array}{l}\text { Regulación de los servicios } \\
\text { públicos }\end{array}$ & \multicolumn{4}{|c|}{$\begin{array}{c}\text { La Autoridad Reguladora de Servicios Públicos (ARESEP) para todas las fases del } \\
\text { subsector eléctrico. }\end{array}$} \\
\hline $\begin{array}{l}\text { Planificación de largo plazo del } \\
\text { subsector eléctrico }\end{array}$ & \multicolumn{4}{|c|}{$\begin{array}{l}\text { El Instituto Costarricense de Electricidad y Telecomunicaciones (ICE) basado en el } \\
\text { plan nacional de energía del MINAE. }\end{array}$} \\
\hline $\begin{array}{l}\text { Centro de control y despacho } \\
\text { del Sistema Eléctrico Nacional } \\
\text { (SEN) }\end{array}$ & \multicolumn{4}{|c|}{ El ICE, con base en criterios técnicos de operación del SEN. } \\
\hline Producción de electricidad & $\begin{array}{l}\text { Generadores } \\
\text { privados }\end{array}$ & Cooperativas & $\begin{array}{l}\text { Empresas } \\
\text { municipales }\end{array}$ & Cogeneradores \\
\hline Transporte de electricidad & \multicolumn{4}{|c|}{ ICE } \\
\hline Distribución & Cooperativas & $\begin{array}{c}\text { Empresas } \\
\text { municipales }\end{array}$ & Compañía & $\begin{array}{l}\text { al de Fuerza y Luz } \\
\text { FL) }\end{array}$ \\
\hline Comercialización & \multicolumn{4}{|c|}{ No se realiza } \\
\hline
\end{tabular}

Fuente: Jiménez G., Roberto, 2009.

\section{OPCIONES DE REFORMA EN EL SECTOR ELÉCTRICO DE COSTA RICA}

Existe en el ámbito internacional una reciente preocupación por el logro de objetivos en el campo social y ambiental que no ha sido posible obtener en el marco de las reformas por la lógica del mercado, aspectos que en el caso del modelo costarricense en gran medida han sido consideradas. No obstante, algunos actores que tienen ciertos intereses y objetivos han mantenido en la agenda de política pública las propuestas de reforma que busca un papel relevante del mercado y una participación abierta de todos los miembros del sector en el mercado eléctrico regional, sin restricciones a la generación privada, como una necesidad pública de relevancia.

El sector eléctrico de Costa Rica ha mostrado un buen desempeño medido a través de algunos indicadores, tal y como se muestra en los cuadros 1 y 2. Lo anterior plantea la necesidad de analizar la conveniencia o no de realizar reformas al sector $y$ si eso fuera necesario, cuál debería ser el marco institucional (reglas del juego, organizaciones, competencias) que permita mantener un sector que aporte al desarrollo del país, en especial manteniendo la solidaridad social, alta cobertura eléctrica y la producción de electricidad con fuentes renovables, como ha sido hasta ahora.

Seguidamente se hace una comparación en dos variables relevantes de países de América Latina que han llevado a cabo reformas en el sector eléctrico con el de Costa Rica, se aclara que existen una gran cantidad de variables que pueden compararse, para efectos de análisis se escogieron estas.

El precio del Kwh para el sector residencial del grupo de países analizados son significativamente más elevados que los de Costa Rica. Países considerados como exitosos en sus procesos de reforma, como Chile, tienen para junio del 2005 precios de 12,34 centavos de US \$/KWh, mientras que Costa Rica tiene 6,69 \$/KWh, es decir un $84,45 \%$ menos que Chile. 


\section{CUADRO 1}

PRECIOS DE LA ELECTRICIDAD DE ALGUNOS PAÍSES DE AMÉRICA LATINA, POR SECTORES DE CONSUMO, EN CENTAVOS DE US\$ POR KWH

\begin{tabular}{ccccccc}
\hline \multirow{2}{*}{ País } & \multicolumn{2}{c}{ RESIDENCIAL } & \multicolumn{2}{c}{ INDUSTRIAL } & \multicolumn{2}{c}{ COMERCIAL } \\
\cline { 2 - 7 } & $01 / 06 / 1996$ & $01 / 06 / 2005$ & $01 / 06 / 1996$ & $01 / 06 / 2005$ & $01 / 06 / 1996$ & $01 / 06 / 2005$ \\
\hline Argentina & 11,91 & 3,79 & 8,36 & 4,67 & 14,65 & 7,07 \\
Brasil & 7,16 & 10,19 & 5,52 & 5,17 & 11,33 & 9,00 \\
Chile & 13,70 & 12,34 & 7,67 & 7,98 & 11,40 & 12,93 \\
Colombia & 4,25 & 9,82 & 8,54 & 8,91 & 10,21 & 11,78 \\
Costa Rica & 7,22 & 6,69 & 10,03 & 7,72 & 11,52 & 8,89 \\
El Salvador & 7,95 & 11,64 & 11,25 & 12,39 & 10,53 & 13,53 \\
Guatemala & 7,06 & 11,70 & 9,56 & 13,69 & 8,08 & 14,56 \\
Nicaragua & 10,66 & 13,60 & 10,03 & 12,46 & 13,33 & 16,53 \\
Panamá & 12,04 & 12,50 & 9,99 & 10,23 & 12,03 & 12,19 \\
Perú & 14,96 & 12,72 & 5,70 & 7,22 & 12,16 & 10,33 \\
América Latina & 9,50 & 8,99 & 5,60 & 6,12 & 10,46 & 10,16 \\
\hline
\end{tabular}

Fuente: Elaboración propia con base en datos de OLADE. Informe de Estadísticas Energéticas. 2005.

Colombia es el país con precios más bajos de los países que han llevado a cabo relevantes reformas con 9,82 us $\$$ por Kwh, teniendo un precio del 46,79\% más altos que los precios del sector residencial de Costa Rica. Es importante ver que el precio de Costa Rica es menor en el sector residencial al precio del promedio de América Latina. Argentina tiene intervenidos los precios.

Se puede apreciar que Costa Rica es el país con mayor proporción de su capacidad instalada con fuentes renovables con un 78,61\%. Unido a ello, la diversificación de fuentes energéticas

\section{CUADRO 2}

CAPACIDAD DE GENERACIÓN ALGUNOS PAÍSES DE AMÉRICA LATINA POR FUENTE ENERGÉTICA EN MW, AÑO 2005

\begin{tabular}{|c|c|c|c|c|c|c|c|c|c|c|c|}
\hline \multirow[b]{3}{*}{ País } & \multicolumn{11}{|c|}{ TIPO DE PLANTA DE GENERACIÓN } \\
\hline & \multicolumn{2}{|c|}{ Hidro } & \multicolumn{2}{|c|}{ Térmica } & \multicolumn{2}{|c|}{ Otras (1) } & \multicolumn{2}{|c|}{ Nuclear } & \multicolumn{2}{|c|}{ Total } & \multirow{2}{*}{$\begin{array}{c}\% \\
\text { Renovables }\end{array}$} \\
\hline & MW & $\%$ & MW & $\%$ & MW & $\%$ & MW & $\%$ & MW & $\%$ & \\
\hline Argentina & 9852,00 & 34,95 & 17288,29 & 61,34 & 26,62 & 0,09 & 1018,00 & 3,61 & 28184,91 & & 35,05 \\
\hline Brasil & 73278,70 & 76,29 & 20770,42 & 21,62 & 0,00 & 0,00 & 2007,00 & 2,09 & 96056,12 & 100,00 & 76,29 \\
\hline Chile & 4812,10 & 39,47 & 7378,40 & 60,52 & 2,00 & 0,02 & 0,00 & 0,00 & 12192,50 & 100,00 & 39,48 \\
\hline Colombia & 8984,27 & 66,88 & 4418,92 & 32,90 & 29,30 & 0,22 & 0,00 & 0,00 & 13432,49 & 100,00 & 67,10 \\
\hline Costa Rica & 1304,05 & 66,48 & 419,57 & 21,39 & 238,08 & 12,14 & 0,00 & 0,00 & 1961,70 & 100,00 & 78,61 \\
\hline El Salvador & 460,90 & 37,42 & 619,70 & 50,31 & 151,20 & 12,27 & 0,00 & 0,00 & 1231,80 & 100,00 & 49,69 \\
\hline Guatemala & 716,90 & 34,29 & 1344,70 & 64,32 & 29,00 & 1,39 & 0,00 & 0,00 & 2090,60 & 100,00 & 35,68 \\
\hline Nicaragua & 104,63 & 13,50 & 582,97 & 75,21 & 87,50 & 11,29 & & 0,00 & 775,10 & 100,00 & 24,79 \\
\hline Panamá & 846,00 & 56,10 & 662,00 & 43,90 & 0,00 & 0,00 & 0,00 & 0,00 & 1508,00 & 100,00 & 56,10 \\
\hline Perú & 3207,06 & 51,40 & 3031,48 & 48,59 & 0,70 & 0,01 & & 0,00 & 6239,24 & 100,00 & 51,41 \\
\hline América Latina & 141152,46 & 54,10 & 113809,90 & 43,62 & 1547,58 & 0,59 & 4390,00 & 1,68 & 260899,93 & & 54,70 \\
\hline
\end{tabular}

(1) Inlcuye energía geotérmica, eólica y solar.

Fuente: Elaboración propia con base en datos de OLADE, Informe de Estadísticas Energéticas 2005. 
brinda al país el primer lugar, seguido de El Salvador, este país concentra en otras fuentes como la geotermia, mientras que en el caso de Costa Rica, cuenta además de esa importante fuente, con la energía eólica y la biomásica. Los países que han llevado a cabo reformas en el sector eléctrico con la excepción de Brasil, tienen una capacidad instalada preponderantemente con fuentes no renovables.

La evolución de la cobertura eléctrica de Costa Rica ha mejorado hasta llegar al año 2008 al 98,6\%, una de las más alta de América Latina.

El sector eléctrico de Costa Rica ha tenido reformas parciales de carácter heterodoxo, que por una parte han mantenido las organizaciones públicas, el sistema verticalmente integrado y una importante regulación de los precios y actividades del sector eléctrico. Se han creado marcos jurídicos especiales para la participación privada en la generación eléctrica $y$ se ha aprobado un convenio marco de mercado eléctrico con Centro América.

En general, con base en indicadores de desempeño se puede decir que el sector eléctrico ha respondido a las necesidades del desarrollo del país de forma bastante positiva. No obstante, existen riesgos $y$ amenazas en este sector como lo son: el rezago en la inversión por limitaciones de gobierno central a las inversiones del ICE, las restricciones para el desarrollo de proyectos renovables por aspectos ambientales para todos los desarrolladores y legales para el caso de los generadores privados, importantes rezagos tarifarios $y$ la potencial competencia con fuentes no renovables en el marco del mercado eléctrico regional.

De lo anterior ha surgido en la agenda pública nacional desde hace más de una década, la necesidad de una reforma al sector eléctrico de Costa Rica. Las posibles implicaciones dependiendo del modelo de reforma son diversos y de una significativa relevancia para prácticamente todos los sectores y ámbitos de la vida del país.

La pregunta que surge es si el tipo de modelo que se formaría, sería mejor al actual y sobre todo si permitirá y creará las condiciones para un desarrollo y operación del sector eléctrico que tenga como hasta ahora una capacidad instalada y generación preponderantemente con fuentes renovables con solidaridad social.

Dentro de las macro propuestas de reforma que se han planteado están las siguientes: (MINAE, 2006; Unión de Cámaras y Asociaciones de la Empresa Privada (UCAAEP), 2005):

a) Mantener el modelo actual, dando mayor libertad de inversión a las empresas públicas.

b) Mantener el modelo actual, dando mayor libertad de inversión a las empresas públicas, además de eliminar las restricciones a las empresas privadas para que puedan generar electricidad con fuentes renovables para el mercado interno específicamente al ICE, con el cual el ICE mantendría la función de comprador único.

c) Mantener el modelo actual, dando mayor libertad de inversión a las empresas públicas, además de eliminar las restricciones a las empresas privadas para que puedan generar electricidad con fuentes renovables para el mercado regulado y no regulado, pudiendo exportar a la región, así el ICE deja de ser comprador único.

d) Efectuar una reforma integral que permita la creación de mercados para las empresas públicas y privadas, competir en el mercado local y centroamericano. Las empresas verticalmente integradas se mantienen; del ICE se separarían las actividades de carácter nacional como la planificación, el Centro de control del despacho y la transmisión de electricidad. Se plantea una disociación por costo de las empresas verticalmente integradas. Modelo apoyado por el Gobierno de Arias y las Cámaras empresariales.

e) Crear mercados competitivos, para lo cual se deben separar las empresas verticalmente integradas, creando nuevas empresas para luego privatizarlas, con el fin de tener suficientes agentes económicos con poco poder en el mercado. Se crearía un nuevo marco legal y organizaciones para ello.

Con base en el análisis realizado por Jiménez (2009), los modelos más posibles son de prospectiva y redes de política, d y e, seguidamente se presentan los efectos más relevantes. 


\section{VIII.LAS IMPLICACIONES ANTE LOS \\ ESCENARIOS MÁS POSIBLES DE REFORMA \\ DEL SECTOR ELÉCTRICO DE COSTA RICA}

El proceso de reformas neoclásicas ha estado liderado por la UCAAEP y los productores privados de electricidad, lo cual ha tenido una congruencia casi total con las propuestas $y$ argumentos del Gobierno de Arias (punto d). Ante el escenario más posible de reforma neoclásica se tienen una serie de efectos sobre el modelo actual, algunos de los cuales se analizan seguidamente.

a. El ICE dejaría de ser el responsable del suministro eléctrico, pasando a las distribuidoras.

b. Difícilmente se podría mantener la planificación normativa existente hasta ahora en el mediano y largo plazo, pasando a ser las señales del mercado, las que determinen en mayor medida las decisiones de inversión e incluso de operación del sistema eléctrico.

c. Pese a que la disociación legal o privatización no se ha planteado a corto plazo, una reforma que crea mercados hará necesaria esas acciones, con el fin de tener en la lógica de las señales de precios, más transparencia $y$ menos poder de mercado de los agentes económicos.

d. Relacionado con lo anterior, necesariamente se deberían dar a mediano plazo la disociación o la privatización para eliminar las empresas verticalmente integradas.

e. El ICE dejaría de ser el comprador único y de tener la responsabilidad del centro de despacho del sistema eléctrico nacional en el mercado nacional y el MER.

f. Se daría una igualación de todas las organizaciones del sector eléctrico, en el nuevo diseño según la reforma más neoclásica, las organizaciones públicas o privadas serían simples operadores del sistema eléctrico, con iguales derechos y obligaciones.

Ante la posible reforma se tienen al menos dos opciones: a. Mantener el sistema regulado y la planificación normativa flexible, que permita definir con precisión la forma cómo se desarrollarán los proyectos de generación y de transmisión de electricidad. b. Dejar que por medio del mercado y las señales de precios, los diferentes agentes económicos decidan en qué, cuándo y cuánto invertir.

Las fuentes renovables convencionales como la hidroeléctrica de filo de agua $y$ de embalse de regulación y la geotermia, han logrado posicionarse, por medio de un proceso de planificación de largo plazo de carácter normativo. Han logrado tener una importante presencia en la capacidad instalada y generación del sistema eléctrico del país. No obstante, surgen dudas que bajo la lógica del mercado se pueda dar la integración de las convencionales y otras fuentes renovables nuevas, cuya necesidad de investigación, desarrollo $y$ viabilidad técnica $y$ financiera requieren de tiempo y recursos, además de instrumentos de política más allá del mercado, que permitan tomar la decisión de invertir; dados los riesgos y que en la etapa de operación sean despachadas al sistema eléctrico.

\section{CONCLUSIONES GENERALES}

Los procesos de reforma en el sector eléctrico en América Latina han tenido resultados ambiguos, la evidencia empírica no muestra que esas transformaciones del sector permitan lograr mejores resultados que lo que Costa Rica con un modelo regulado, empresas verticalmente integradas y realizando labores de planificación de largo plazo y despacho del sistema eléctrico por medio del ICE, han podido conseguir hasta ahora.

La consideración del marco institucional expresado en organizaciones fuertes con capacidad de gestión para ejercer la rectoría, planificar a largo plazo, ejecutar, supervisar, evaluar $y$ regular, con leyes, normas y procedimientos claros y respetados por todos, son elementos fundamentales para que cualquier sistema eléctrico pueda funcionar bien. En el caso de Costa Rica el modelo actual ha permitido pese a sus debilidades un buen desempeño. Por lo anterior, debe justificarse muy bien una posible reforma, siendo cautos con las diferentes alternativas que se analicen.

En una política nacional que priorice los renovables, si el mercado es el que define qué 
invertir y qué plantas se despachan, dominarán aquellas fuentes de menor costo, de recuperación más rápida, de menor riesgo, con más energía firme, en donde las renovables y en especial las fuentes nuevas tienen menor posibilidad de desarrollarse.

La participación pública y privada en un sistema mixto regulado $y$ heterodoxo propio de las condiciones del entorno costarricense $y$ de ciertos valores que la sociedad ha ido creando parece ser el mejor camino, por encima de modelos estandarizados en el ámbito internacional según la perspectiva neoclásica. Esto permitirá asegurar el suministro eléctrico como hasta ahora, mantener la solidaridad social y sobre todo la generación con fuentes renovables en una alta proporción como ha sido hasta ahora. Los espacios para la participación privada son necesarios, pero acotados de acuerdo con la política de largo plazo en donde el Estado define y orienta el desarrollo y operación del sistema eléctrico, por medio de planificación normativa, la regulación de tarifas y la venta de la electricidad a un comprador único responsable del suministro eléctrico nacional.

\section{BIBLIOGRAFÍA}

Alcantara, Manuel. Gobernabilidad, crisis y cambio. México: Fondo de Cultura Económica, 1995.

Bacon, R. \& Besants, J. "Global electric power reform, privatization, and liberalization of the electric power industry in developing countries". Revista anual de Energía y Ambiente 26. Banco Mundial, 2001.

Banco Mundial. Informe sobre el desarrollo mundial, 1997: El Estado en un mundo en transformación. Washington DC. 1997.

Bazán Navarro, Ciro. "Efectos de la reforma del sector eléctrico: modelización teórica y experiencia internacional". [Tesis para optar por el grado de Doctorado en
Economía]. Universidad de las Palmas de Gran Canaria, 2003.

Beder, S. \& Cahill, D. "Neo-Liberal think tanks and neo-liberal restructuring: Learning the lessons form proyect Victoria and the privatization of Victoria's electricity industry". Social Alternativas 1(24). First quarter. 2005.

Besant Jones, J. "Reforming Power Markets in Developing Countries: What have we learned?" World Bank Paper 19. World Bank, setiembre, 2006.

Besant, J. \& Tenenbaum, B. "Enseñanzas de la crisis de energía de California". Revista Finanzas y Desarrollo. Washington. 2001.

CEPAL. Estrategia para el fomento de las fuentes renovables de energía en América Central. LC/MEX/L.620. 2004.

Cortina, Adela. Ciudadanos del mundo: Hacia una teoría de la ciudadanía. Madrid: Alianza Editorial, 1998.

Dussán, Manuel. "Nicaragua: Opciones de política para la reforma del sector eléctrico". Informe técnico. Dpto. de desarrollo sostenible. Nueva York: Banco Interamericano de Desarrollo (BID), 2004.

Estache, Antonio. “Cómo hacer que las reformas de la infraestructura en América Latina favorezcan a los pobres?". Revista CEPAL 78, diciembre. 2002.

Ffrench, R. Development and External Debt in Latin America: Bases for a New Consensus. R. Ffrench \& R. Feinberg (Eds.). Indiana: University of Notre Dame Press, 1988.

Germa, B. "Estado ¿versus? Mercado". Revista de Economía aplicada 2 (22). España. 2004. 
Hodgson, Geoffrey. "El enfoque de la economía institucional". Revista Comercio Exterior 10 (53). México. 2003.

Instituto Costarricense de Electricidad. Plan de Expansión de la generación eléctrica. San José, Costa Rica. 2008.

Jamasb, Tooraj. "Between the state and market: Electricity sector reform in developing countries". Utilities Policy 14. UK: Faculty of Economics, University of Cambridge, 2006.

Jiménez Gómez, Roberto. "Análisis del proceso de formulación de políticas para la reforma del sector eléctrico y su potencial incidencia en el marco institucional $y$ la producción con fuentes renovables". [Tesis sometida para optar al grado de Doctor en Gobierno y Políticas Públicas]. Universidad de Costa Rica, abril, 2009.

Krugman, Paul. "Vendiendo prosperidad". Ariel Sociedad Económica. España. 2000.

Millán, Jaime. Entre el mercado y el Estado: tres décadas de reformas en el sector eléctrico de América Latina. Nueva York: BID, 2006.

MINAE. Informe del proceso de formulación del modelo de la industria eléctrica de Costa Rica. San José, Costa Rica. 2006.

North, Douglas. Estructura y cambio en la historia económica. Madrid: Alianza Universidad, 1984.
North, Douglas. "Desempeño económico en el transcurso de los años". Conferencia dictada al recibir el premio Nobel de Economía. Estocolmo, Suecia. 1993.

North, Douglas. Institututions, Institucional change and economic perfomance. (Reimpresión 18). Cambridge: University Press, 2005.

Pollitt, Michael G. "Electricity Reform in Chile: Lessons for Developing Countries". En: Cambridge Working Papers in Economics CWPE 0448. Massachusetts Institute of Technology, Center for Energy and Environmental Policy Research, University of Cambridge, Department of Applied Economics. 2005.

Stiglitz, Joseph. "El rumbo de las reformas. Hacia una nueva agenda para América Latina". Revista CEPAL 80. Santiago de Chile. 2003a.

Stiglitz, Joseph. Los felices 90. La semilla de la destrucción. Argentina: Editorial Taurus, 2003b.

Unión Costarricense de Cámaras y Asociaciones de la Empresa Privada (UCCAEP). "Modernización y fortalecimiento del sistema eléctrico nacional". Mimeógrafo. 2005.

Valdivieso, Susana. "North y el cambio histórico: luces y sombras de la Nueva Historia Institucional". Revista de Economía Institucional 4. Colombia. 2001. 